\title{
Association Between Faculty and Student Self-Efficacy and Attitudes Toward Evidence-Informed Practice
}

\author{
Veronica Foster \\ St. Olaf College \\ Joshua Bernstein \\ A.T. Still University
}

This study examined the association between faculty self-efficacy and attitudes toward teaching evidenceinformed practice (EIP) and student self-efficacy and attitudes toward applying EIP. Novel instruments were developed and implemented. Forty-two faculty members and 121 students participated. No significant associations between faculty and students were found, and students would prefer more practical EIP assignments or opportunities to apply EIP in more meaningful ways. EIP-related curriculum at the institution may be more important than faculty self-efficacy and attitude or previous experience. Although faculty must be trained to teach EIP, focusing efforts on EIP-related curriculum may be more important.

Keywords: curriculum, evidence informed practice, evidence based medicine, health professions education, integrative medicine, higher education, self efficacy

\section{INTRODUCTION AND BACKGROUND}

The use of evidence-informed practice (EIP) is increasingly considered a professional responsibility and a desired clinical competency in most health professions (Thomas, Han, Osler, Turnbull, \& Douglas, 2017). Application of EIP in clinical settings has been demonstrated to "improve the quality and reliability of health care, enhance patient outcomes, and reduce costs for the U.S. health care system" (Levin \& Feldman, 2012, p. xv). Heightened awareness of the benefits of EIP in clinical practice led to development of EIP curricula in health profession educational programs to improve the EIP-related knowledge, skills, behaviors, and attitudes of students (Thomas et al., 2017). EIP is currently included in professional education programs for medicine (Liabsuetrakul, Sirirak, Boonyapipat, \& Pornsawat, 2013), pharmacy (Neill \& Johnson, 2012), nutrition and dietetics (Hinrichs, 2018), occupational therapy (Thomas et al., 2017), speech-language therapy (Spek, Wieringa-de Waard, Lucas, \& van Dijk, 2013), chiropractic medicine (Haas, Leo, Peterson, LeFebvre, \& Vavrek, 2012), nursing (Levin \& Feldman, 2012), athletic training (A.T. Still University [ATSU], 2018), and a variety of other health professions.

As EIP becomes integrated into health professions education, the need to evaluate the effectiveness of curriculum strategies and teaching methods will increase. Although self-efficacy and attitude toward applying EIP has been studied in clinical (Bussières et al., 2015; Malik, McKenna, \& Plummer, 2015; Roecker, Long, Vining, \& Lawrence, 2013; Schneider et al., 2015; Sullivan, Leach, Snow, \& Moonaz, 2017), faculty (Abdelkarim \& Sullivan, 2014; Anderson, Kligler, Taylor, Cohen, \& Marantz, 2014; Bury, 
2011), and student populations (Abdelkarim \& Sullivan, 2014; Spek et al., 2013; Thomas et al., 2017), no study to date has examined the relationship between faculty self-efficacy and attitude toward teaching EIP and student self-efficacy and attitude toward applying EIP at an integrative health sciences (IHS) university. EIP is considered a best practice in health care (Levin \& Feldman, 2012; Zwickey et al., 2014) and many IHS institutions are integrating EIP into curricula (Thomas et al., 2017; Zwickey et al., 2014). Even with the increase in prevalence, few studies have examined the relationship between faculty and student selfefficacy and attitudes in IHS programs.

For the purpose of this study, EIP is defined as the incorporation of evidence to guide clinical decision making (Northwestern Health Sciences University [NWHSU] and Regents of the University of Minnesota [UM], 2011). EIP incorporates the current best evidence from research including randomized controlled trials, descriptive and qualitative research, case reports, and expert opinion; a patient's presentation, preferences, and values; a clinician's experience; and the history of the profession. The terms evidencebased practice and evidence-based medicine are also frequently used with similar meaning.

For this study, self-efficacy was defined as an individual's perception of the ability to successfully perform a behavior. Attitude is the extent to which a person feels positively or negatively about engaging in a specific behavior and is commonly considered to result from the individual's evaluation of the behavior and belief that performing a behavior will lead to certain outcomes (DiClemente, Crosby, \& Kegler, 2009).

Application refers to the use of EIP principles in clinical practice or research for clinical decisionmaking and informing clinical practice (NWHSU and Regents of the UM, 2011). Although teaching EIP may include some elements of application, teaching and applying EIP are distinctly different in scope, intention, and use. This is an important delineation because self-efficacy and attitude toward applying EIP may not be equivalent to self-efficacy and attitude toward teaching EIP.

Teaching EIP to students may include instruction of EIP courses, administration of EIP activities in the classroom, assignment of EIP activities outside of the classroom, modeling EIP application in the classroom or clinic environment, consistently using research-related terminology and language, online tutorials, journal clubs, and incorporating research evidence into course notes or other materials, clinical or scientific discussions, and other activities related to clinical decision making (National Center for Complementary and Integrative Health, 2017; Zwickey et al., 2014).

The purpose of this research was to examine the effects of faculty self-efficacy and attitude toward teaching EIP on student self-efficacy and attitude toward applying EIP at an IHS institution. By gaining a better understanding of this relationship, IHS institutions will make effective curricular changes and support faculty in meaningful ways to facilitate learning and EIP competence in students. Furthermore, because an instrument did not exist to measure the constructs defined in the present study, a novel instrument was developed by the researchers. This instrument may be useful at IHS institutions and facilitate better assessment of student-faculty interactions related to EIP.

\section{SIGNIFICANCE OF THE PROBLEM}

Bandura (1993) suggested a teacher's self-efficacy for motivating and promoting learning affects the types of learning opportunities teachers create for students and the level of academic achievement of students. Self-efficacy and attitude toward EIP may influence whether faculty choose to incorporate EIP activities into courses and how information is presented by faculty may influence students' self-efficacy, attitude, and future application of EIP in clinical practice (Lee, 2007; Schrader \& Brown, 2008; Tsui, 2001). Positive interactions with faculty are important for the self-efficacy and academic success of ethnic minority students who may not have a supportive social network to achieve academic goals (DeFreitas \& Bravo, 2012). Academic success of ethnic minority students in healthcare programs is vital to improve the longstanding inequity of workforce representation (Betancourt, Beiter, \& Landry, 2013) and increase the cultural sensitivity of patient care (Degrie et al., 2017). Faculty enthusiasm and self-efficacy for teaching, creativity, and experimentation are necessary to cultivate critical thinking skills in students. Faculty attitudes, beliefs, and assumptions influence students' critical thinking skills, and low faculty confidence may also be a detriment to student self-efficacy (Myall, Levett-Jones, \& Lathlean, 2008; Tsui, 2001). 
Individuals with similar knowledge and skills frequently perform differently depending on self-efficacy and the influence and relationship between faculty and students must be considered (Bandura, 1993). Bandura (1993) stated, "teachers' beliefs in their personal efficacy to motivate and promote learning affect the types of learning environments they create and the level of academic progress their students achieve" (p. 117). If faculty incorporate EIP changes to courses without competency using this technique, they may develop low self-efficacy and a poor attitude toward teaching EIP. This may impair the quality of EIP information and skills delivered to students (Schonert-Reichl, 2017; Tsui, 2001). Furthermore, SchonertReichl (2017) and Tsui (2001) proposed faculty attitude and level of stress are linked to development of critical thinking and level of stress in students, which may lead to low self-esteem, anxiety, and behavioral problems.

Bandura (1993) emphasized the effect of faculty perception and attitude are so potent that faculty belief in their collective teaching efficacy has greater influence on school-level achievement than the effects of student body characteristics. Student-faculty interactions have a considerable role in occupational decisions, academic aspirations and success, and student development of competence and sense of purpose (Lamport, 1993; Lee, 2007; Myall et al., 2008). Given the current understanding of the influence of faculty on student learning, it is necessary to examine the effects of faculty self-efficacy and attitude toward teaching EIP on student self-efficacy and attitude toward applying EIP.

\section{METHODS}

This study required sampling from two populations at an IHS institution, faculty and students. All ranked and adjunct faculty at the IHS university during the time of the study $(\mathrm{N}=99)$ across six departments were eligible and invited to participate (see Table 1).

TABLE 1

FACULTY EMPLOYED BY DEPARTMENT

\section{Faculty Employed by Department}

\begin{tabular}{llll}
\hline Department & Ranked & Adjunct & Total \\
Chiropractic & 29 & 10 & 42 \\
Acupuncture/Chinese Med. & 10 & 12 & 22 \\
Nutrition & 1 & 2 & 3 \\
Massage Therapy & 7 & 8 & 15 \\
Undergraduate & 7 & 6 & 13 \\
Clinical Education & 1 & 3 & 4 \\
Total Faculty & 55 & 44 & 99 \\
\hline
\end{tabular}

Note. Although a member of the faculty may be active in more than one department, each faculty member is represented only once

All students who were enrolled in at least one course at the IHS university during data collection $(\mathrm{N}=$ 918) were eligible and invited to participate (see Table 2). Trimesters represent a student's progress in the program with $\mathrm{T} 1$ representing a student's first trimester. Because the undergraduate program does not follow a standardized cohort schedule like the other programs, students were divided into postbaccalaureate in pre-health (PBPH), Bachelor of Science in human biology (BS), and non-degree seeking students. 
TABLE 2

STUDENTS ENROLLED BY DEPARTMENT

\begin{tabular}{|c|c|c|c|c|c|c|c|c|c|c|c|}
\hline Program & T1 & $\mathrm{T} 2$ & $\mathrm{~T} 3$ & $\mathrm{~T} 4$ & T5 & T6 & $\mathrm{T} 7$ & T\& & TO & T10 & Totol \\
\hline Chiropractic & 142 & 0 & 53 & 131 & 0 & 54 & 91 & 0 & 44 & 89 & 604 \\
\hline Acu./Chinese Med. & 21 & 0 & 21 & 12 & 0 & 10 & 16 & 1 & 9 & $\mathrm{n} / \mathrm{a}$ & 90 \\
\hline Massage Therapy & 27 & 12 & 11 & 13 & $\mathrm{n} / \mathrm{a}$ & $\mathrm{n} / \mathrm{a}$ & $\mathrm{n} / \mathrm{a}$ & $\mathrm{n} / \mathrm{a}$ & $\mathrm{n} / \mathrm{a}$ & $\mathrm{n} / \mathrm{a}$ & 63 \\
\hline & \multicolumn{3}{|c|}{ PBPH } & BS & & \multicolumn{3}{|c|}{ Non-degree } & \multicolumn{3}{|c|}{ Total } \\
\hline $\begin{array}{l}\text { Undergraduate } \\
\text { Total Students }\end{array}$ & \multicolumn{3}{|l|}{129} & 28 & & \multicolumn{3}{|c|}{1} & \multicolumn{3}{|c|}{161} \\
\hline
\end{tabular}

Note. Insufficient data for nutrition students. $\mathrm{T}=$ Trimester.

To the researchers' knowledge, no existing survey instrument captures the self-efficacy and attitudes of faculty toward teaching EIP or compares these constructs with students applying EIP. Because no existing survey instrument fit the needs of this study, the EIP Teaching and Learning (EIP-TL) scale was developed, validated, and implemented. Two versions of the scale were developed: the EIP-TL Faculty Version and the EIP-TL Student Version. A scoring rubric was also developed.

Before administering the EIP-TL scale to the study populations, the validity and reliability were assessed. To determine face and content validity, two EIP education experts reviewed the instrument and assessed whether the items on the questionnaire will measure the intended constructs, and are readable, inclusive, and clear. Because assessment of criterion-related validity depends on availability of another instrument or predictor, criterion-related validity cannot be assessed (Bolarinwa, 2015). Feedback and recommendations provided by the two EIP education experts were incorporated into the instrument.

Next, reliability of the instrument was assessed using a pilot-test with a small group of faculty and students at another IHS university located in the Midwest. Prior to data collection, approval was obtained from the IRB committees at the pilot-test institution and the institution under study. Using Cronbach alpha, internal consistency reliability of the EIP-TL scale was analyzed. Evaluation of the validity and reliability assessments following the pilot-test informed a final revision of the instrument to be implemented with the study population.

This study included a single data collection period using two surveys (one for each study population). Surveys were delivered electronically to collect data from faculty and students over a 4-week period. At the start of the 4-week period, survey links were emailed using the electronic platform, SurveyMonkey, to all faculty and students who met the study criteria. A total of three reminder emails were sent. One reminder email was sent per week during weeks $2-4$ of the data collection period.

\section{RESULTS}

Of the 99 faculty members and 918 students invited to participate, 42 faculty members $(42.4 \%)$ and 121 students (13.2\%) completed the surveys. The EIP-TL scales were scored according to the scoring rubric. Although scores for individual constructs differed slightly between faculty and students, total scores were nearly identical between groups (see Table 3). Students' scores for intention to apply EIP in future clinical practice were $24.3 \%$ higher than faculty members' scores for intention to teach EIP in the classroom or clinical settings. Faculty members' scores for self-efficacy in teaching EIP were $18.1 \%$ higher than students' scores for self-efficacy in applying EIP clinically. 
TABLE 3

EIP-TL SCALE SCORES

\begin{tabular}{|c|c|c|c|}
\hline Construct & Faculty Mean & Student Mean & Difference \\
\hline$\overline{\text { Attitude }}$ & 10.48 & $\overline{10.42}$ & $\overline{0.06}$ \\
\hline Intention & 5.43 & 7.17 & -1.74 \\
\hline Self-efficacy & 7.57 & 6.20 & 1.37 \\
\hline Total & 23.48 & 23.69 & -0.21 \\
\hline
\end{tabular}

Cronbach's alpha was used to analyze the internal consistency and reliability of the EIP-TL scale. The Cronbach's alpha coefficients were acceptable ( 0.7 or greater) for all construct subtotals and final totals except student attitude (0.586), student intention (0.548), and faculty attitude (0.509). Since there were only 3-4 items for each construct, the Cronbach's alpha coefficients for the total scores $(0.721$ for faculty and 0.786 for students) may be more meaningful.

Associations between faculty and student subscores and total scores were analyzed using Pearson $r$, ANOVA tests, and independent samples $T$-tests. Several Pearson $r$ coefficients were significant. The study group (faculty or student) was associated with intention $(p<.01)$ and self-efficacy $(p<.02)$ scores. Attitude scores were associated with intention $(\mathrm{p}<.01)$, self-efficacy $(\mathrm{p}<.01)$, and total scores $(\mathrm{p}<.01)$. Intention and self-efficacy scores were associated with total scores $(\mathrm{p}<.01)$. There were no statistically significant associations between faculty scores and student scores.

ANOVA tests were used to analyze associations between construct and total scores and demographic items. For faculty members, a greater number of years spent at the institution was associated with higher attitude scores $(\mathrm{p}<.05)$ and experience with EIP before joining the institution was associated with higher intention scores $(p<.05)$. The intention to teach EIP score was significant in the librarian group $(p<.05)$ and the score for self-efficacy in teaching EIP was significant in the researcher group $(p<.05)$. The programs in which a faculty member taught and the highest level of completed education were not significantly associated with construct or total scores.

For students, experience with EIP before joining the institution was associated with attitude $(p<.02)$, self-efficacy $(\mathrm{p}<.001)$, and total scores $(\mathrm{p}<.002)$. The intention to apply EIP score was associated with students' progress in the program $(\mathrm{p}<.05)$, with students toward the end of their programs exhibiting higher intention scores. Full- or part-time status, time at the institution, program of study, and highest level of completed education were not significantly associated with construct or total scores.

There was no statistically significant association between faculty and student self-efficacy $(p=.477)$ and faculty and student attitudes $(p=.374)$. When the faculty and student groups were segmented by health profession program, the only significant association was between faculty attitude and student self-efficacy in the undergraduate program $(\mathrm{p}<.001)$. No other comparisons reached the required level of significance.

There were no significant differences between programs in student self-efficacy and attitude toward applying EIP in clinical practice, or between self-efficacy and attitude and type or field of the highest educational degree in both faculty and students.

One open-ended question on each instrument asked, "What do you think can be done to improve the use of EIP at your institution?" The constant comparative method (CCM) for qualitative data analysis was used to analyze this item. Twenty-one faculty participants shared ideas in response to this item. Using the $\mathrm{CCM}$, responses were grouped into categories, resulting in four themes: ideas for incorporating EIP in the classroom or clinic, ideas for EIP-related changes to the curriculum, ideas for EIP-related changes to the culture of the institution, and ideas for faculty development.

Faculty ideas for incorporating EIP in the classroom or clinic included increasing use of case studies, creating more opportunities for students to use EIP, and modeling EIP use. For example, one participant recommended having "students research a condition of their patient's and apply the evidence to their treatment plan". Most ideas for EIP-related changes to the curriculum were related to moving the EIP course to the first trimester of each program and promoting a stronger thread of EIP topics in all courses in the 
curriculum through strong leadership and oversight. One faculty member wrote, "meaningful curriculum review and oversight; making sure faculty leaders are engaged in it" as a way to improve the use of EIP at the institution. Ideas for change to institutional culture included concerns about the focus on EIP at the institution and existing policies and dogma that are perceived as excessive. A participant wrote, "I'm concerned that the school is more interested in research, rather than patient preference and the doctor's clinical experience". Ideas for faculty development included training faculty on how to use EIP in a classroom setting, how to find and read quality clinical literature, how to summarize and interpret key findings to convey to others, and how to determine study quality. One participant requested that administrators "train faculty on how to use EIP in a classroom setting".

Thirty-three student participants responded to this item and responses were grouped into seven categories: EIP education should be more meaningful or practical, EIP education could be made more clear or basic, EIP should be taught more consistently throughout the curriculum, specific ideas for activities, EIP is used too much at the institution, issues with instructors, and general comments that do not include EIP-related ideas.

Several students commented that they would prefer more practical EIP assignments or opportunities to apply EIP in more meaningful ways. One student wrote "bring up current research articles based on topics covered in the course to give a 'life application' to the material that we are learning. Learning facts is fine, but learning how to apply them is way more valuable and important for practice and educating patients". Three participants expressed confusion or insecurities about how EIP is taught in different classes and suggested that EIP be made more clear or fundamental when applied in different courses. A participant wrote, "I'm still learning. More research sources and easy access to them" would improve the student's EIP learning experience. Participants expressed a desire for others to filter the research and provide them with appropriate research evidence to consider in practice. A popular theme was the need for consistency in EIP education throughout the curriculum. One student highlighted that "certain classes do not require the usage of EIP due to the content of the class, but it would benefit from the usage of [EIP] for further consolidating the information relayed to the student". Several students asked for a more consistent thread of EIP throughout all courses to provide varied and repetitive practice in the context of different subjects.

The most frequent type of response included specific ideas for EIP activities. Suggestions included focusing on how standards of practice change based on particular studies, practice and tips for optimizing search results, or searching for EIP treatment options for every condition covered in classroom presentations. Three participants shared that they think EIP is too heavily relied upon at the institution and would prefer to emphasize other elements of patient care. One student wrote, "science changes too quickly and therefore should not be the driving force in a healing profession. Once humans understand what heals the body as much as the intelligence that maintains the body, then and only then will science-led practice be beneficial. This is not saying EIP is not important - it is merely putting it in its proper place". Four responses were related to issues with instructors in specific courses. These comments included statements like "the instructor needs to know what they are teaching" and assertions that the school should hire "more qualified instructors." The final category of responses included general comments that offered no suggestions for improvement such as, "there are so many poorly conducted research [studies] that seem to be conducted well at a quick glance. When it comes to the body the cause of most joint dysfunctions are multifactorial. Many researchers fail to understand this simple concept and thus conduct research focusing on only one of the factors and end up with poor results, then publish the article saying X is not effective...". Participants shared their surprise about things they have learned from research evidence, frustration about poorly conducted research, and other comments unrelated to EIP.

\section{CONCLUSIONS}

The results of this study have practical significance for educators and administrators. First, the selfefficacy and attitude of faculty toward teaching EIP is not a strong determinant of students' self-efficacy and attitude toward applying EIP in clinical practice at this IHS institution. This finding may contradict previous evidence that interactions with EIP-capable instructors are important for students' self-efficacy 
and attitude toward EIP (Boström et al, 2018; Hinrichs, 2018; Thomas et al., 2017). It is possible that inclusion of EIP-related topics and activities throughout the curriculum is more relevant than individual faculty member's self-efficacy and attitude toward teaching EIP. Previous investigators found teachers' beliefs strongly influenced classroom practices and willingness to incorporate topics and activities in the classroom (Organisation for Economic Co-operation and Development, 2009; Robinson \& Hope, 2013; Salem \& Jones, 2010; Tsui, 2001). Given the importance of EIP-related topics and activities throughout the curriculum, it is interesting that faculty self-efficacy and attitude toward EIP was not a stronger determinant of student self-efficacy and attitude toward EIP at this institution. It is possible that willingness to incorporate EIP-related topics and activities is more closely related to self-efficacy teaching EIP than to teachers' attitude toward EIP. Although faculty members generally report a positive attitude toward EIP inclusion in curricula, many are reluctant to include EIP-related activities due to lack of self-efficacy and other barriers (Anderson et al., 2014; Salem \& Jones, 2010; Young, Esterhuizen, Volmink, \& Clarke, 2016).

Second, no difference in self-efficacy and attitude exists among the students in different programs. This may indicate that students at this IHS institution are receiving the same fundamental skills and emphasis on EIP regardless of program specialization. The chiropractic, acupuncture and Chinese medicine, and massage therapy programs include courses on fundamentals of EIP that are adjusted to the educational level of each program. Although nutrition and undergraduate health sciences programs do not include specific EIP courses, EIP is heavily emphasized in courses throughout the curricula of all programs at the institution. Even though the volume, depth, and timeframe of EIP-related education vary between the programs, there was no difference in self-efficacy and attitude of students. It is possible that the strong institutional culture surrounding the importance of EIP facilitates students' interest and use of EIP.

Third, the type and field of a participant's highest educational degree obtained were not associated with self-efficacy or attitude. This finding was interesting because significant variation of academic experience exists among faculty and student participants in the different programs. Even though faculty and students in the doctoral programs had more previous educational experience than those in the master's, undergraduate, and certificate programs, this did not significantly affect self-efficacy or attitude. The EIPrelated training at the IHS institution for both faculty and students may be effective to the extent that previous educational experience is not required. Participants' previous education may not have included significant training or emphasis on EIP, even when they reported having some experience with EIP before entering the institution under study.

Collectively, EIP-related curriculum at the IHS institution may be more important to student selfefficacy and attitude toward applying EIP than faculty self-efficacy and attitude toward teaching EIP or previous educational experience. Although faculty must be sufficiently trained to teach EIP, focusing efforts on inclusion of EIP-related activities throughout the curriculum may be important. When students were given the opportunity to suggest ways to improve the use of EIP in the classrooms or clinics at the IHS institution, 27 out of 38 responses included ways that EIP could be better included throughout the curriculum. Improvements to EIP-related activities and elements of curriculum may have a greater effect on student self-efficacy and attitude than efforts to improve faculty self-efficacy and attitude. Previous investigators suggest students need access to EIP learning activities and strategies to prepare for real-world clinical use of EIP and students perceive opportunities to use EIP as dependent on clinical instructors (Boström et al, 2018; Hinrichs, 2018; Thomas et al., 2017). Faculty self-efficacy and attitude toward teaching EIP must be sufficient to provide EIP learning activities, but the relationship between student and faculty self-efficacy and attitude appears to be limited at the institution under study.

The results of this study may be explained in part by consistently high EIP self-efficacy and attitude among faculty. Previous curricular changes at this IHS institution to implement EIP may explain the lack of significance of faculty self-efficacy and attitudes. A significant EIP training program for faculty was implemented that included EIP education, opportunities to practice EIP teaching strategies, and was sensitive to the needs and interests of faculty members (Evans et al., 2011; Kreitzer et al., 2008; Zwickey et al., 2014). The extensive education and support available to faculty contributed to an institutional culture of EIP inclusion in aspects of curriculum whenever possible. Because faculty members at this IHS 
institution generally reported high self-efficacy and attitude toward teaching EIP, this may have minimized the apparent importance of these contributions.

Considering the limitations of this study is necessary when reviewing the conclusions stated above. Although no significant relationships among groups were detected, the results may have been different with a higher student response rate or with larger subgroups within each population. Furthermore, because participants were recruited from a single IHS institution, generalizing these results to other student or faculty groups should be done with care. The strong institutional culture of EIP may have minimized the importance of the relationship between faculty and students. In the present study, it appears that student self-efficacy and attitude toward EIP are independent of faculty influences, but this may be unique to this institution because of the strong institutional culture emphasizing the importance and use of EIP.

\section{RECOMMENDATIONS}

The following are recommendations for members of faculty and administration to improve EIP education in HPEP. Further research is also recommended to expand on the results of the present study.

\section{Faculty}

EIP is considered a responsibility and desired clinical competency in most health professions (Thomas et al., 2017; Zwickey et al., 2014), so it is important for faculty to collaborate with other stakeholders to successfully implement EIP throughout health professions curricula. The results of this study demonstrate faculty self-efficacy and attitude toward teaching EIP do not significantly affect a student's self-efficacy and attitude toward applying EIP at this IHS institution. EIP-related topics and activities delivered throughout the curriculum may be most important. Faculty members should offer opportunities whenever possible for students to review foundational principles of EIP, practice EIP application, and transfer EIP skills learned in the classroom into a real-world setting. Previous investigators suggest students perceive these opportunities as useful to success in future clinical practice (Hinrichs, 2018; Thomas et al., 2017). Faculty members without experience using or teaching EIP should request training, activities, and other support from administrators to make EIP-related course changes.

\section{Administrators}

The findings of previous studies support the need for EIP-related elements to be consistently implemented across curricula. Student comments in this study and previous investigations have highlighted the need for diverse EIP-related learning activities offered frequently in multiple courses throughout a health profession educational program (Hinrichs, 2018; Thomas et al., 2017). The effort made by administrators at this IHS institution to foster a culture of EIP inclusion may explain the lack of statistical significance of faculty self-efficacy and attitudes. Administrators at the IHS institution under study had previously implemented a continuous EIP training program for faculty, which likely increased the selfefficacy and attitudes of faculty members at the institution (Evans et al., 2011; Kreitzer et al., 2008; Zwickey et al., 2014). The extensive education and support available to faculty contributed to an institutional culture of EIP inclusion, which may explain consistently high EIP self-efficacy and attitude among faculty. Administrators in health profession educational programs should provide faculty members with education, support, and resources to carry out curricular changes to increase EIP inclusion.

\section{Researchers}

Based on the results in this study, additional research is recommended. First, EIP research should be repeated at other health profession educational institutions to determine whether results are applicable to a more diverse population. Second, other determinants of student self-efficacy and attitude toward EIP could be evaluated. Characteristics like volume, depth, or timeframe of EIP curricular elements could be measured to determine their effects on student self-efficacy and attitude toward EIP. Finally, other elements of student success using EIP may be studied including barriers, culture of an educational institution, culture of a health profession, risk perception, and more. 


\section{REFERENCES}

Abdelkarim, A., \& Sullivan, D. (2014). Perspectives of dental students and faculty about evidence-based dental practice. Journal of Evidence-Based Dental Practice, 14(4), 165-173. https://doi.org/10.1016/j.jebdp.2014.06.001

Anderson, B.J., Kligler, B., Taylor, B., Cohen, H.W., \& Marantz, P.R. (2014). Faculty survey to assess research literacy and evidence-informed practice interest and support at Pacific College of Oriental Medicine. Journal of Alternative \& Complementary Medicine, 20(9), 705-712. https://doi.org/10.1089/acm.2014.0138

A.T. Still University. (2018). Applications by program. Retrieved from https://www.atsu.edu/contact/apply

Bandura, A. (1993). Perceived self-efficacy in cognitive development and functioning. Educational Psychologist, 28(2), 117-148. Retrieved from https://www.uky.edu/ eushe2/Bandura/Bandura1993EP.pdf

Betancourt, J.R., Beiter, S., \& Landry, A. (2013). Improving quality, achieving equity, and increasing diversity in healthcare: The future is now. Journal of Best Practices in Health Professions Diversity: Education, Research \& Policy, 6(1), 903-917. Retrieved from https://www.uncpress.org/journals/journal-of-best-practices-in-health-professions-diversity/

Bolarinwa, O.A. (2015). Principles and methods of validity and reliability testing of questionnaires used in social and health science researches. Nigerian Postgraduate Medical Journal, 22(1), 195-201. https://doi.org/10.4103/1117-1936.173959

Boström, A.M., Sommerfeld, D.K., Stenhols, A.W., \& Kiessling, A. (2018). Capability beliefs on, and use of evidence-based practice among four health professional and student groups in geriatric care: A cross-sectional study. PLoS ONE, 13(2), 1-15. https://doi.org/10.1371/journal.pone.0192017

Bury, S. (2011). Faculty attitudes, perceptions and experiences of information literacy: A study across multiple disciplines at York University, Canada. Journal of Information Literacy, 5(1), 45-64. https://doi.org/10.11645/5/1/1/513

Bussières, A.E., Terhorst, L., Leach, M., Stuber, K., Evans, R., \& Schneider, M.J. (2015). Self-reported attitudes, skills and use of evidence-based practice among Canadian Doctors of Chiropractic: A national survey. The Journal of The Canadian Chiropractic Association, 59(4), 332-348. Retrieved from https://www.chiropractic.ca/jcca-online/

DeFreitas, C.S., \& Bravo, A., Jr. (2012). The influence of involvement with faculty and mentoring on the self-efficacy and academic achievement of African American and Latino college students. Journal of the Scholarship of Teaching and Learning, 12(4), 1-11. Retrieved from http://scholarworks.iu.edu/journals/index.php/josotl/article/view/2083

Degrie, L., Gastmans, C., Mahieu, L., Dierckx de Casterlé, B., \& Denier, Y. (2017). How do ethnic minority patients experience the intercultural care encounter in hospitals? A systematic review of qualitative research. BMC Medical Ethics, 18(1), 1-17. https://doi.org/10.1186/s12910-016-01638

DiClemente, R.J., Crosby, R.A., \& Kegler, M.C. (2009). Emerging theories in health promotion, practice, and research (2nd edition). San Francisco, CA: John Wiley \& Sons, Inc.

Evans, R., Delagran, L., Maiers, M., Kreitzer, M.J., \& Sierpina, V. (2011). Advancing evidence informed practice through faculty development: The Northwestern Health Sciences University Model. Explore (NY), 7(4), 265-268. https://doi.org/10/1016/j.explore.2011.04.014

Haas, M., Leo, M., Peterson, D., LeFebvre, R., \& Vavrek, D. (2012). Evaluation of the effects of an evidence-based curriculum on knowledge, attitudes, and self-assessed skills and behaviors in chiropractic students. Journal of Manipulative and Physiological Therapeutics, 35(9), 701-709. https://doi.org/10.1016/j.jmpt.2012.10.014 
Hinrichs, R.J. (2018). Dietetic interns' perceptions and use of evidence-based practice: An exploratory study. Journal of the Medical Library Association, 106(1), 65-73. https://doi.org/10.5195/jmla.2018.308

Kreitzer, M.J., Sierpina, V., Maiers, M., Delagran, L., Baldwin, L., Evans, R., \& Chase, M. (2008). Ways of knowing: Integrating research into CAM education and holism into conventional health professional education. Explore, 4(4), 278-281. https://doi.org/10.1016/j.explore.2008.04.012

Lamport, M.A. (1993). Student-faculty interaction and the effect on college student outcomes: A review of the literature. Adolescence, 28(112), 971-990. Retrieved from https://www.journals.elsevier.com/journal-of-adolescence/

Lee, C.J. (2007). Academic help seeking: Theory and strategies for nursing faculty. The Journal of Nursing Education, 46(10), 468-475. Retrieved from https://www.ncbi.nlm.nih.gov/labs/journals/j-nurs-educ/

Levin, R., \& Feldman, H.R. (2012). Teaching evidence-based practice in nursing (2nd edition). New York City, NY: Springer Publishing Company.

Liabsuetrakul, T., Sirirak, T., Boonyapipat, S., \& Pornsawat, P. (2013). Effect of continuous education for evidence-based medicine practice on knowledge, attitudes and skills of medical students. Journal of Evaluation in Clinical Practice, 19(4), 607-611. https://doi.org/10.1111/j.1365-2753.2012.01828.x

Malik, G., McKenna, L., \& Plummer, V. (2015). Perceived knowledge, skills, attitude and contextual factors affecting evidence-based practice among nurse educators, clinical coaches and nurse specialists. International Journal of Nursing Practice, 21, 46-57. https://doi.org/10.1111/ijn. 12366

Myall, M., Levett-Jones, T., \& Lathlean, J. (2008). Mentorship in contemporary practice: The experiences of nursing students and practice mentors. Journal of Clinical Nursing, 17(14), 1834-1842. https://doi.org/10.1111/j.1365-2702.2007.02233.x

National Center for Complementary and Integrative Health. (2017). NCCIH Facts-at-a-glance and mission. Retrieved from https://nccih.nih.gov/about/ataglance

Neill, K.K., \& Johnson, J.T. (2012). An advanced pharmacy practice experience in application of evidence-based policy. American Journal of Pharmaceutical Education, 76(7), 1-7. https://doi.org/10.5688/ajpe767133

Northwestern Health Sciences University and Regents of the University of Minnesota. (2011). Evidence informed practice. Retrieved from https://www.csh.umn.edu/research/foundations-evidenceinformed-practice-modules

Organisation for Economic Co-operation and Development. (2009). Creating effective teaching and learning environments: First results from TALIS. Paris: OECD. Retrieved from https://www.oecd.org/education/school/43023606.pdf

Robinson, T.E., \& Hope, W.C. (2013). Teaching in higher education: Is there a need for training in pedagogy in graduate degree programs? Research in Higher Education Journal, 21, 1-11. Retrieved from https://www.aabri.com/rhej.html

Roecker, C.B., Long, C.R., Vining, R.D., \& Lawrence, D.J. (2013). Attitudes toward evidence-based clinical practice among Doctors of Chiropractic with diplomate-level training in orthopedics. Chiropractic \& Manual Therapies, 21(43), 1-7. https://doi.org/10.1186/2045-709X-21-43

Salem, L., \& Jones, P. (2010). Undaunted, self-critical, and resentful: Investigating faculty attitudes toward teaching writing in a large university writing-intensive course program. Writing Program Administration, 34(1), 60-83. Retrieved from https://www.wpacouncil.org/archives/34n1/34n1 salem-jones.pdf

Schneider, M.J., Evans, R., Haas, M., Leach, M., Hawk, C., Long, C., . . Terhorst, L. (2015). US chiropractors' attitudes, skills and use of evidence-based practice: A cross-sectional national survey. Chiropractic \& Manual Therapies, 23(1), 1-12. https://doi.org/10.1186/s12998-0150060-0 
Schonert-Reichl, K.A. (2017). Social and emotional learning and teachers. Future of Children, 27(1), 137-155. Retrieved from http://www.jstor.org/stable/44219025

Schrader, P.G., \& Brown, S.W. (2008). Evaluating the first year experience: Students' knowledge, attitudes, and behaviors. Journal of Advanced Academics, 19(2), 310-343. https://doi.org/10.4219/jaa-2008-775

Spek, B., Wieringa-de Waard, M., Lucas, C., \& van Dijk, N. (2013). Teaching evidence-based practice (EBP) to speech-language therapy students: Are students competent and confident EBP users? International Journal of Language \& Communication Disorders, 48(4), 444-452. https://doi.org/10.1111/1460-6984.12020

Sullivan, M., Leach, M., Snow, J., \& Moonaz, S. (2017). Understanding North American yoga therapists' attitudes, skills and use of evidence-based practice: A cross-national survey. Complementary Therapies in Medicine, 32, 11-18. https://doi.org/10.1016/j.ctim.2017.03.005

Thomas, A., Han, L., Osler, B.P., Turnbull, E.A., \& Douglas, E. (2017). Students' attitudes and perceptions of teaching and assessment of evidence-based practice in an occupational therapy professional Master's curriculum: A mixed methods study. BMC Medical Education, 17(1), 64. http://doi.org/10.1186/s12909-017-0895-2

Tsui, L. (2001). Faculty attitudes and the development of students' critical thinking. The Journal of General Education, 50(1), 1-28. Retrieved from http://www.jstor.org/stable/27797860

Young, T., Esterhuizen, T.M., Volmink, J., \& Clarke, M. (2016). Attitude and confidence of undergraduate medical programme educators to practice and teach evidence-based healthcare: A cross-sectional survey. International Journal of Evidence-Based Healthcare, 14(2), 74-83. http://doi.org/10.1097/XEB.0000000000000068

Zwickey, H., Schiffke, H., Fleishman, S., Haas, M., Cruser, D.A., LeFebvre, R., . . Gaster, B. (2014). Teaching evidence-based medicine at complementary and alternative medicine institutions: Strategies, competencies, and evaluation. Journal of Alternative \& Complementary Medicine, 20(12), 925. https://doi.org/10.1089/acm.2014.0087 